\title{
Objective Assessment Tools: Physical Parameters in Scar Assessment
}

M. E. H. Jaspers and P. Moortgat

\section{Contents}

\subsection{Clinimetrics - 150}

17.2 Color -151

17.2.1 Erythema and Pigmentation - 151

17.2.2 Reflectance Spectroscopy - 151

17.2.3 Spectrophotometric Analysis (SIA) - 152

17.3 Elasticity -153

17.3.1 Cutometer -153

17.3.2 DermaLab - 155

17.3.3 Tonometers -155

17.4 Perfusion - 156

17.4.1 Laser Doppler Imaging - 156

17.4.2 Laser Speckle Imaging - 156

17.5 Conclusion - 157

References - 157 


\section{Background}

Several types of problematic scars can be identified: hypertrophic scars, keloids, contractures, and adherent scars (• Fig. 17.1). All these scars require specialized treatment. However, the need for innovation and novel treatment is paramount to further reduce the burden of these scars and to ultimately attain scarless healing.
Critical to the development of novel treatment modalities are objective assessment tools that evaluate whether scar treatment is effective and successful. Secondly, assessment tools can be used to monitor the scar's response to interventions. One important aspect to take into account when using tools in clinical practice is to ascertain that the chosen tool is "clinimetrically approved."
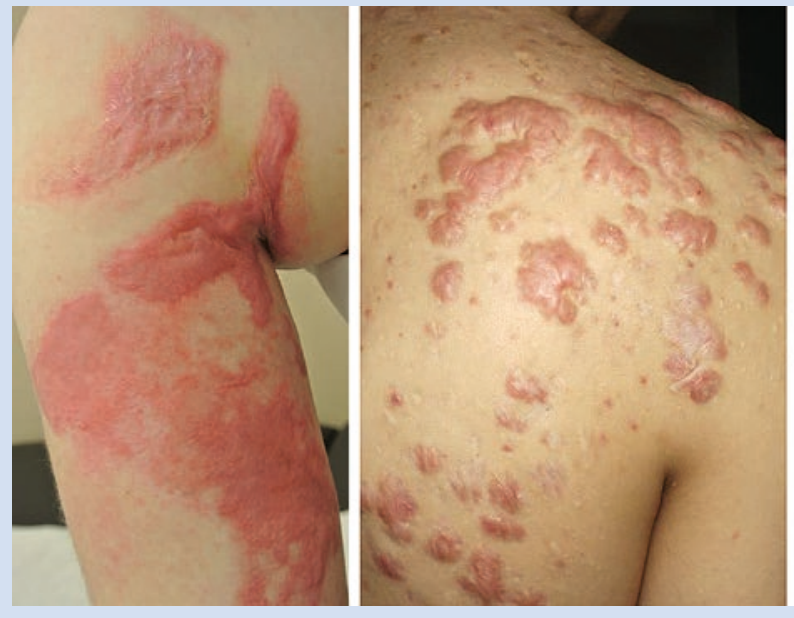

- Fig. 17.1 Several types of pathological scars. a Hypertrophic scar on the right upper arm, b keloids on the right scapula and shoulder, c contracture of the right axilla, and $\mathbf{d}$ widespread adherent scarring on a patient's back and spine. (Reproduced
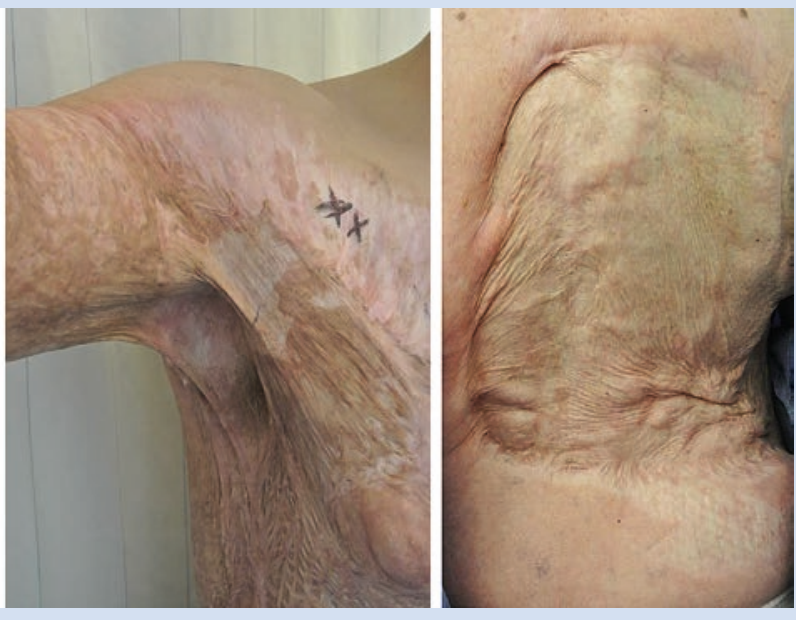

with permission from 'Beyond the skin, new insights in burn care"” by M. E. H. Jaspers, 2017. ISBN: 978-94-6233-852-4. (C) All rights reserved)

\subsection{Clinimetrics}

The discipline of "clinimetrics" (i.e., measurement in medicine) aims to improve the quality of measurements by assessment of the properties of existing tools or by development of new tools. Before implementation of a new measurement tool in either clinical practice or in research can be considered, two essential properties need to be evaluated: reliability and validity. All measurement tools are required to produce reliable and valid scores.

The general definition of reliability is "the degree to which the measurement is free from measurement error" $[1,2]$. In addition, there is an extended definition of reliability: "the extent to which scores for patients who have not changed are the same for repeated measurements under several conditions," which makes clear that repeated measurements are a key point. The variation that may arise between repeated measurements decreases the reliability. This can be attributed either to the measurement tool, the persons performing the measurement, the patients undergoing the measurement, or the circumstances under which the measurements are performed.
The measurement error comprises both the systematic and random error of a patient's score that cannot be attributed to true changes in the scar. So, parameters of measurement error are relevant for the measurements of changes in scar status. Moreover, these parameters are of great value for clinicians as they are expressed in the units of measurement, which facilitates interpretation. Finally, to determine whether changes are clinically relevant, the minimally important change (MIC) can be calculated, which is defined as "the smallest change in score which patients, clinicians or relevant others perceive as important" $[2,3]$. To monitor the scar of an individual patient, it is of paramount importance that the MIC is smaller than the measurement error of the tool.

Validity is defined as "the degree to which an instrument truly measures what it purports to measure" [1]. Validity can be divided into three types: content validity, construct validity, and criterion validity. Content validity focuses on the correspondence of the content of the measurement tool with the construct that is intended to measure. Content validity is assessed qualitatively during development by pretesting, expert opinion, and literature review. Construct validity is applicable in situ- 
ations in which there is no gold standard, and therefore this type of validity refers to whether the measurement tool provides the expected scores, based on knowledge about the construct. Criterion validity focuses on the correspondence of the (new) measurement tool with the gold standard (i.e., criterion). In theory, the gold standard is a perfectly valid assessment, but this rarely exists in practice. Also in scar care, it is challenging to identify a suitable criterion. In light of this chapter and of using tools in the future, it is important to take this into account and to be aware of the reliability and validity of the gold standard or comparator instrument itself.

Over the years, the number of available tools has increased rapidly. However, it is important to realize that not every scar parameter can be assessed and that the use of technologies and corresponding terminology is not standardized yet. Hereby, an overview is provided for three important physical scar parameters that can be assessed by (noninvasive) objective tools: color, elasticity, and perfusion.

\subsection{Color}

\subsubsection{Erythema and Pigmentation}

When looking at hypertrophic scars, one could ask the question: Which feature makes these scars so obvious and therefore problematic? Besides increased thickness or an irregular surface, the amount of erythema and pigmentation appear to have a major impact on the judgment of scar quality by both clinicians and patients. Moreover, the assessment of erythema could be imperative to predict the end of the remodeling phase [4]. Therefore, color measurements are an essential part of scar evaluation, and the associated measurement outcomes are common parameters in scar research.

It is beyond the scope of this chapter to state the underlying causes of the formation of erythema and pigmentation. However, it is important to realize how these aberrant scar features are constituted, to consequentially assess them in the right way. Moreover, it is underlined that the terminology regarding color measurements is not standardized yet. In clinical practice and in research, the measurement outcomes blood flow, scar color, erythema, and redness are used interchangeably or often replaced by the umbrella term "vascularization," which is not appropriate [5].

For the purpose of clarity, it is beneficial to provide a detailed description of every feature when assessed by a certain tool. Erythema is a complex characteristic that may be defined as the level of oxygenated hemoglobin measured at $660 \mathrm{~nm}$, which shows to have a correlation with subjectively assessed redness [5]. Furthermore, scar color is also constituted by the amount of pigmentation, expressed by the outcome measurement melanin.

\subsubsection{Reflectance Spectroscopy}

Reflectance spectroscopy has been in use for over 60 years to assess skin color. At present, many affordable handheld devices are available that can easily be used in daily clinical settings. The most commonly used principles for measuring scar color are tristimulus reflectance colorimetry and narrow-band reflectance spectrophotometry. Both principles determine color by measuring the intensity of reflected light of specific wavelengths. The main chromophores in human scar tissue, hemoglobin and melanin, are primary determinants of the color. It has to be taken into account that these chromophores can be measured separately, but may influence each other. It is suggested that a scar with high hemoglobin values can mask the amount of melanin and vice versa.

\subsubsection{Tristimulus Colorimetry}

Tools based on the principle of tristimulus reflectance colorimetry are the Chroma Meter CR-400 (Konica Minolta Sensing Inc., Osaka, Japan) and the SkinColorimeter CL 400 (Courage and Khazaka electronic $\mathrm{GmbH}$, Köln, Germany). This principle was developed to objectively represent color in a manner analogous to how color is perceived by the human eye. As well as with narrow-band spectrophotometry, white light is sent to the skin by LEDs in a probe. Light is scattered in all directions whereas the light reflected from the skin or scar is measured through three particular wavelength filters and expressed accordingly. This technique expresses XYZ-values, which can be calculated into RGB (red/ green/blue) values or the related three $\mathrm{L} * \mathrm{a} * \mathrm{~b}$ components: $\mathrm{L} *$ (brightness), a* (amount of red-green), and $b *$ (amount of yellow-blue) [6, 7]. These components are based on the color system of the Commission International d'Eclairage (CIE) Lab system.

Concerning the clinimetric properties, the parameter LAB 2 by the Colorimeter shows and ICC of 0.95 for the interrater reliability [6]. The Chroma Meter was first investigated on healthy skin, showing ICC values $>0.92$ for all parameters $(L *, a *$, and $b *)$ during measurements by two observers, thereby reporting the interrater reliability [8]. The latter study also reports standard error of measurement (SEM) values, which are between 0.38 and 0.59 and thereby low. In addition, a preceding type of the Chroma Meter (CR-221) was evaluated on scar tissue and shows ICC values $>0.73$ for a single measure- 
ment by one observer (test-retest) and ICC values $>0.91$ when the measurements of four observers are averaged [9]. In this study, SEM values are between 1.26 and 4.33, which is rather high.

\subsubsection{Narrow-Band Spectrophotometry}

Narrow-band tools are based on the fact that hemoglobin and melanin show different spectral curves for the absorption of light. The tools offer read-out of erythema and melanin values as well as CIE $\mathrm{L} * \mathrm{a} * \mathrm{~b}$ values. The erythema and melanin index values are based on the differences in light absorption of red and green by hemoglobin and melanocytes, respectively. The erythema index is defined as: $E=100 \times \log$ (intensity of reflected red light/intensity of reflected green light) and the melanin index can be defined as: $\mathrm{M}=100 \times \log (1 /$ intensity of reflected red light). It has to be taken into account that the index values can be measured separately, but may influence each other.

In the DSM III ColorMeter (Cortex Technology, Hadsund, Denmark) this is achieved by two lightemitting diodes (LEDs) (green $568 \mathrm{~nm}$ and red $655 \mathrm{~nm}$ ) that illuminate a surface and record the intensity of reflected light using a photodetector. The Mexameter MX 18 (Courage and Khazaka electronic GmbH, Köln, Germany) uses 16 light-emitting diodes that emit light at three wavelengths (green: $568 \mathrm{~nm}$; red $660 \mathrm{~nm}$; infrared $880 \mathrm{~nm}$ ), where after the reflected light is measured by a receiver unit. Erythema is measured by the green and red wavelengths, whereas melanin is determined by the red and infrared wavelengths. Readings are expressed between 0 and 1000, corresponding to white and black respectively.

Both tools are examined in several clinical studies and provide reliable data on scars [10]. The most common reliability parameter examined is the intraclass correlation coefficient (ICC) for ratings performed by several observers (i.e., interrater reliability). Erythema measured by the Mexameter shows an ICC between 0.82 and 0.97 and for the DSM II ColorMeter an ICC of 0.84 is reported $[6,11]$. In addition, the DSM II ColorMeter also offers read-out of CIE $\mathrm{L} * \mathrm{a} * \mathrm{~b}$ values, which will be described in the next paragraph. Investigation of the parameter $\mathrm{a} *$ by the DSM II ColorMeter shows an ICC of 0.94 [6]. Unfortunately, information on parameters concerning the measurement error of these color tools is lacking. Furthermore, attempts are made to assort the most valid parameter of each tool [6]. However, as previously mentioned, it is often difficult to appoint an adequate comparator instrument that exactly measures the same feature (i.e., construct) of a scar as the tool under study. This is underscored by the poor correlations reported between color-measuring tools and scar assessment scales [10,
12]. Overall, the correlation between erythema assessed by a tool and redness assessed by a scar assessment scale is between 0.4 and $0.7[5,11,12]$.

Ultimately, the DermaLab Combo (Cortex Technology, Hadsund, Denmark) will be mentioned here. This device offers a combination of measurement possibilities. The color probe uses the principle of reflectance spectrometry to measure scar color and all abovementioned parameters can be reproduced: erythema, melanin, $\mathrm{L} *, \mathrm{a} *$, and $\mathrm{b} *$. As previously stated, the parameters erythema and $a *$ can be used to indicate scar erythema. A weak to moderate correlation is found between erythema values and redness scored by a subjective tool and in terms of reliability, the ICC varies between 0.66 and 0.84 for measurements by two observers, whereas the test-retest reliability is even lower: $0.29-0.42[13,14]$.

Several studies show that erythema index scores decline over time $[15,16]$ and all clinical studies report that distinction between scar tissue and healthy skin can be made with color-measuring tools. Therefore, it is stated that objective color measurements appear reliable to determine change over time in a (heterogeneous) study population, which merits their scientific use. However, for the clinical follow up of an individual patient, SEM values need to be very low. Moreover, concerning the validity, it is challenging to define the exact measured construct, which is becoming even more complex given that the tools can reflect various parameters. This has to be taken into account when a color-measuring tool is selected to monitor the scar's response to interventions.

From a practical point of view, it is important to notice that pressure on the scars will change the color toward the white spectrum. Therefore, users are required to lightly put a color-measuring tool on the scar to reduce the influence of pressure. Another limitation, which also accounts for elasticity-measuring tools, is the small size of the probe's measurement area. To comprehensively present the color or elasticity of a scar as a whole, the proposed solution is to work with a predefined algorithm, covering several measurement areas within the borders of one scar. For example, by drawing an imaginary line through the horizontal and vertical axis of the scar, resulting in five measurement points: one on the intersection of both lines and four halfway the intersection and the border of the scar. In this way, also selection bias by the observer is avoided.

\subsubsection{Spectrophotometric Analysis (SIA)}

To provide information on color outside the visible spectrum, relating to deeper structures of the scar, infrared light can be used. Spectrophotometric intracutaneous 
analysis (SIA) can be performed via a clinical device, the SIAscope, which utilizes a probe that exerts radiation ranging from 400 to $1000 \mathrm{~nm}$ and produces 8 narrowband spectrally filtered images of the skin. These images are processed by software algorithms and allow subsequently visualization and quantification of blood, collagen, and melanin. It already has been used to monitor changes in scar tissue in response to treatment [17].

\subsection{Elasticity}

The second physical parameter that causes poor functional scar quality is a loss of elasticity, which is due to increased collagen synthesis and lack of elastin in the dermal layer. However, as will be emphasized in this paragraph, certain tools quantify the opposite of elasticity, which is tissue hardness.

\subsubsection{Cutometer}

The Cutometer MPA-580 is designed to measure elasticity (i.e., elevation) of the upper skin or scar layer using a predefined negative pressure setting, which mechanically deforms the skin. Negative pressure (i.e., vacuum) is instantly created in the device where after the skin is drawn into the aperture of the probe (• Fig. 17.2). Subsequently, after a defined time, the skin is released. Inside the probe, the vertical deformation of the skin is determined by a noncontact optical measuring system. This optical measuring system consists of a light source and a light receptor and two prisms facing each other that project the light from transmitter to receptor. The light intensity varies due to the penetration depth of the skin. The resistance of the skin to the negative pressure (rigidity) and its ability to return to its original position (elasticity) are displayed as curves (penetration depth in $\mathrm{mm} /$ time) in real time during the measurement. This measurement principle allows data collection on the elastic and mechanical properties of skin or scar surface and enables objective quantification.

The Cutometer is a feasible device containing a probe, which is available in several aperture sizes $(2,4$, 6 and $8 \mathrm{~mm} \varnothing$ ) to fit different skin sites and different study requirements (- Fig. 17.2). The smaller apertures mainly measure the elastic properties of the epidermis. With a larger aperture $(6$ and $8 \mathrm{~mm})$, the measurement also comprises the dermal component, which is designated for scar assessment. As with the color-measuring tools, it is important to ensure light contact is made between the scar and the probe to avoid alterations in outcome measure. Measurements can be monitored as live curves on the screen of a connected laptop. The software of the Cutometer MPA 580 allows calculation of many parameters of interest from the different portions of the measurement curve. The two most relevant parameters are labelled as $\mathrm{R}$ and $\mathrm{U}$ (• Figs. 17.3 and 17.4).

R-Parameters:

- R0: Behavior of the skin/scar to force (rigidity), maximum amplitude of the curve in $\mathrm{mm}$

- R1/R4: Ability of the skin/scar to return to its original state, minimum amplitude after relaxation

- R2: Viscoelasticity: Resistance to mechanical force versus ability of returning in $\%$

- R3/R9: Tiring effect (fatigue) visible with repeated suction/relaxation

- R5: Net elasticity: Elastic portion of the suction part versus the elastic portion of the relaxation part in $\%$
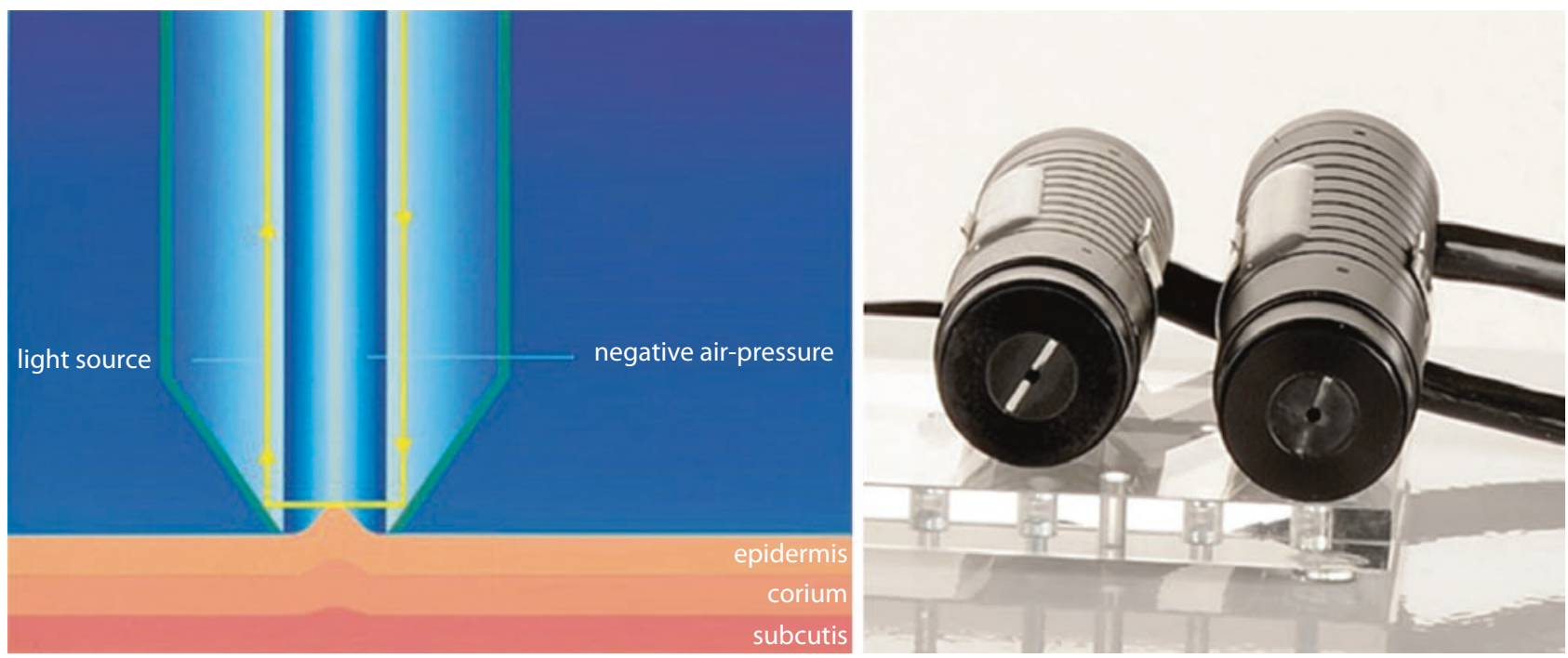

- Fig. 17.2 Left: measuring principle of the Cutometer. Right: different aperture sizes of the probes. Reused with permission from Courage + Khazaka electronic GmbH. (C) All rights reserved 
- Fig. 17.3 The R-parameters of the Cutometer. Reused with permission from Courage + Khazaka electronic $\mathrm{GmbH}$.

(C) All rights reserved
- Fig. 17.4 The U-parameters of the Cutometer
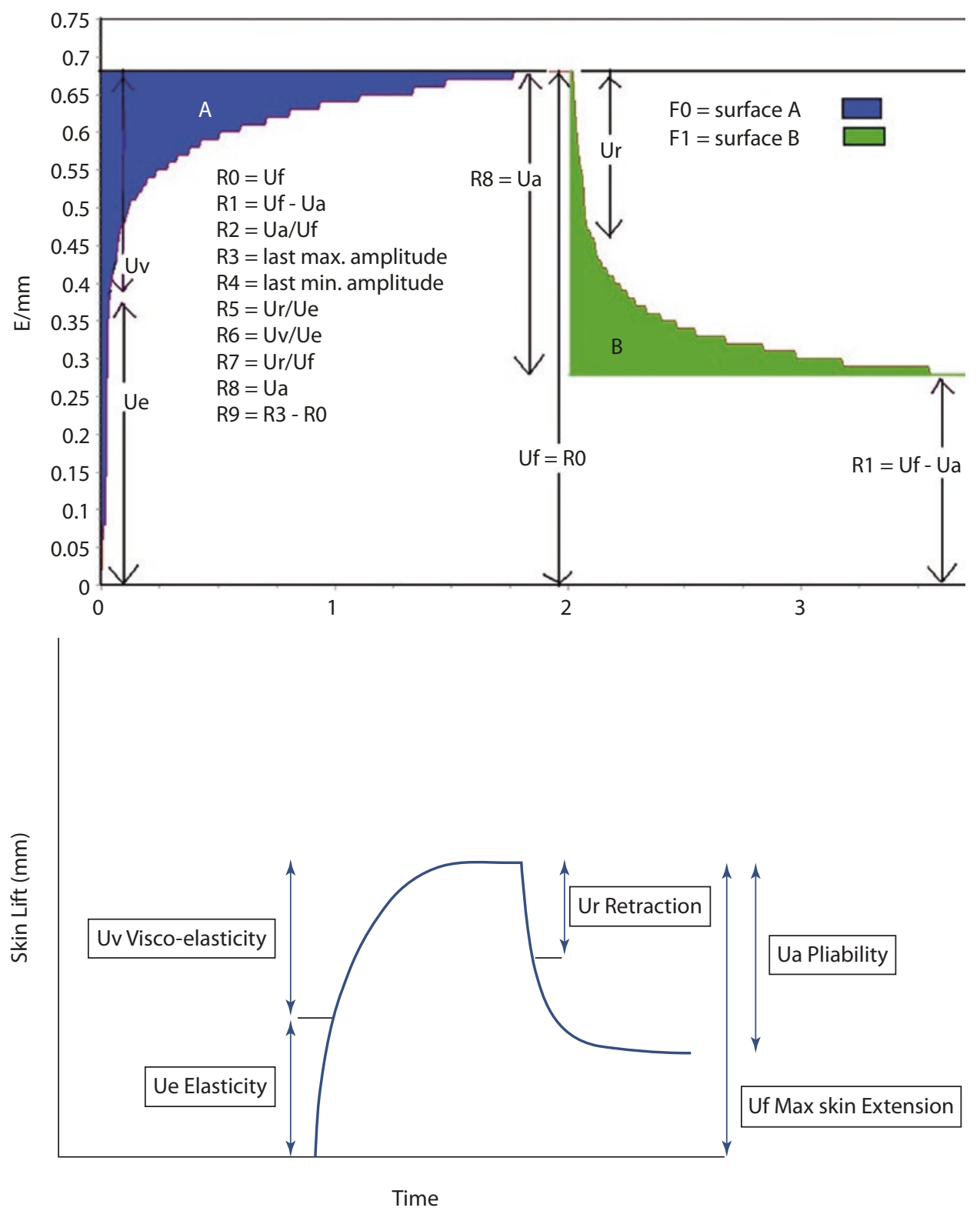

- R6: Portion of the viscoelasticity of the curve in $\%$

- R7: Portion of the elasticity compared to the complete curve in $\%$

- R8: Complete relaxation after the pressure is removed in $\mathrm{mm}$

U-Parameters:

- Uf (maximal skin extension): Deformation at the end of the vacuum period

- Ue (elasticity): Extent of skin stretching within the first $0.1 \mathrm{~s}$ of the vacuum period

- Ua (pliability): Difference between the maximum deformation and the deformation after $1 \mathrm{~s}$ of normal pressure
- Ur (retraction): Relaxation of the skin within the first $0.1 \mathrm{~s}$ after the ending of the vacuum, that is, the ability of the skin to return to its initial position after deformation and is related to the function of elastic fibers

- Uv (viscoelasticity): Difference between the deformation after the $0.1 \mathrm{~s}$ and the maximal deformation

The different parameters and their terms (i.e., elasticity, skin extension, pliability, retraction, and viscoelasticity) suggest that each parameter measures a different aspect of skin or scar deformation. However, the parameters are highly correlated when tested on scars, hence, a sin- 
gle parameter is sufficient to use in the evaluation of scar elasticity (see below) [18].

\subsubsection{Clinimetric Properties}

The reliability of a single measurement performed by a single observer is good for extensibility (Uf) and elasticity (Ue) and ranges $r=0.74-0.76$. For viscoelasticity, pliability, and retraction the reliability is low to moderate: $r=0.35-0.69$. The Coefficient of Variation $(C V=$ S.E.meas $/$ mean $\times 100$ ) for measurements on scars, based on the measurements of four observers, shows the lowest variation for extensibility $(22.5 \%)$, whereas the highest variation is calculated for viscoelasticity measurements $(36.0 \%)$ [18]. The concurrent validity was estimated by calculating the correlation between subjective evaluation of pliability and each of the Cutometer parameters. The correlations were moderate $(r>0.46)$ and statistically significant for each parameter except for viscoelasticity for which the correlation was low.

In general, it is stated that the reliability of the $2 \mathrm{~mm}$ probe is worse than the reliability of larger probes, because the small probe only reflects low values (small skin deformation). This is easily explained by the fact that the ICC or correlation is highly influenced by a wide range in measured values and by a heterogeneous population. So, when higher values or a range of values within a population are measured, this positively affects reliability as expressed by relative parameters. Of all parameters, either maximal skin extension (R0/Uf) or elasticity (Ue) appears to be influenced the least by variations in force applied to the probe and therefore it is the most reliable parameter to detect change in scar elasticity over time or after treatment.

\subsubsection{DermaLab}

The DermaLab elasticity probe (Cortex Technology, Hadsund, Denmark), which is the successor of the Dermaflex, consists of a light plastic probe that is much smaller than that of the Cutometer. This probe is attached to the skin using double-sided adhesive rings to form a closed chamber. Within this chamber, two narrow beams of light run at different heights parallel to the skin surface and serve as elevation detectors. A controlled vacuum is created in the closed chamber in over 30-60 s. In contrast to the Cutometer, the DermaLab probe measures the amount of suction required (in megapascal, MPa) to achieve an elevation of the skin of $1.5 \mathrm{~mm}$. In pronounced adherent scars, this may cause a problem, as the scar is sometimes too stiff to be elevated sufficiently to reach the level of the detectors. The DermaLab shows an excellent ICC for test-retest reli- ability on scars (0.76-0.91); however, on healthy skin the ICC is much lower (ICC 0.45) [14].

Both the DermaLab and the Cutometer show advances of being a "hub," to which other measurement probes can be attached. The DermaLab Combo, for example, provides several probes, considering nine skin parameters (e.g., elasticity, color, transepidermal water loss, temperature), including ultrasound measurement of dermal scar thickness.

\subsubsection{Tonometers}

Tonometry works by exerting pressure on the skin and therefore quantifies predominantly firmness or hardness of tissue. Two types of tonometers can be distinguished: one type is based on air that flows through the system, which is blocked at a certain pressure (e.g., the Tissue Tonometer), and the second type provides an indentation load in the vertical direction (e.g., the Durometer). The Tissue Tonometer (Flinders Biomedical Engineering, Adelaide, Australia) is a weight-loaded device that exerts pressure directly on the area to be measured. The weight drives a blunt probe into the tissue with a tissue deformation as a result. This tissue deformation can be measured in millimeters, with a sensitivity of $0.01 \mathrm{~mm}$. The readings are directly proportional to the firmness of the tissue being measured.

The Tissue Tonometer shows good intra-observer reliability, but a moderate correlation $(r=0.44-0.46)$ with the pliability score of a subjective scoring system (the VSS scale) is found. Additionally, the measurement requires a contralateral reference point, making it a relative measure [Lye 2006]. Another study, using a modified Tissue Tonometer, shows excellent interobserver reliability $(\mathrm{ICC}=0.95)$ for healthy skin, accompanied by a low SEM of $0.025 \mathrm{~mm}$ [19]. A significant difference between scars and normal tissue was also demonstrated in the latter study. The Durometer (Rex Gauge Company, Inc., Glenview, Ill.) also shows good ICCs for the interobserver reliability $(0.82-0.91)$ in one study, however, this is on sclerodermal skin [20].

Important to mention is that tonometers are influenced by the hardness of underlying tissue, which may limit the compression of the skin. This has to be taken into account when measuring locations where bone structures are situated directly under the area of interest. Possibly, measurements in these areas are not reflecting the condition of the skin/scar but instead of the underlying structure. Another shortcoming of tonometers include the need to place the device accurately, which must be within $5^{\circ}$ of upright position to measure correctly. 


\subsection{Perfusion}

Hypertrophic scars result from a wide array of derailed wound healing processes. It is suggested that new blood vessel formation, mainly apparent as angiogenic sprouting of preexisting blood vessels, is an essential process in the development of hypertrophic scars. Therefore, several treatment regimens (e.g., laser, pressure garments, and cryotherapy) work by destructing the microvasculature and/or reducing the blood flow. This may result in hypoxia that would lead to fibroblast degeneration and collagen degradation, both enhancing shrinkage of the hypertrophic scar tissue. As a result, it is of interest to measure scar blood flow (i.e., perfusion).

\subsubsection{Laser Doppler Imaging}

Laser Doppler imaging, a noninvasive flow measurement technique, can be used to quantify and visualize blood flow in scars $[15,21]$. Laser Doppler imaging can be divided in the older technique, laser Doppler flowmetry (LDF), in which the fiber optic probe is in contact with the tissue, and the newer laser Doppler imaging (LDI) devices. The working mechanism is based on the Doppler principle. In LDF, a single-point measure of the cutaneous blood flow in a scar is obtained. LDF systems are therefore more limited compared to the other systems and unsuitable to use in larger heterogeneous scars. However, there are currently modern and high performance LDF systems such as the moorVMS-LDF (Moor Instruments Ltd., Axminster, United Kingdom) or the PeriFlux 5000 (Perimed AB, Järfälla, Sweden) that are ideal for laboratory or scientific use. In LDI, laser light directed at moving erythrocytes in sampled tissue exhibits a frequency change, which is photo detected and processed to generate a line-by-line color-coded map that is proportional to the amount of perfusion in the tissue. After completion of a LDI measurement, data can be analyzed offline using software to calculate the blood flow automatically, where after it is expressed in perfusion units (PU).

To assess blood flow in a scar, the moorLDI Imaging System (Moor Instruments Ltd., Axminster, United Kingdom) can be used. Previous studies show that hypertrophic scars sustain an elevated blood flow compared to normal skin $[15,22]$. Another clinical study also presents a statistically significant increase in blood flow values of scars compared to healthy skin [5]. In more detail, the latter study shows increased LDI blood flow values in 29/32 included patients. Interestingly, this study also compares the association between the outcomes of LDI and colorimetry using the DSM II
ColorMeter. The results indicate that blood flow and erythema values are not correlated. During measurements of 32 hypertrophic scars, a widespread in erythema values is observed, whereas the accompanying blood flow values are mostly beneath $300 \mathrm{PU}$ and thereby low. These data are in agreement with a previous study, presenting that erythema (measured using the skin-colorimeter expressing $\mathrm{L} * \mathrm{a} * \mathrm{~b}$ index values) is associated with blood flow, but this correlation is not consistent at different test moments [4]. As a result, it is concluded that LDI and colorimetry are non-interchangeable measurement tools.

LDI can be a valuable adjunct in the research setting, where it might be useful to monitor scar development and/or response to treatment that is directed at lowering blood flow. However, although new laser Doppler instruments became available over the last years, it is important to keep in mind that the tool has some limitations in terms of feasibility (e.g., high cost, long assessment time, and moderate ease of use). Therefore, LDI seems less suitable for the follow-up of scars in daily practice.

\subsubsection{Laser Speckle Imaging}

Laser speckle imaging (LSI) or laser speckle perfusion imaging (LSPI) are alternative perfusion monitoring techniques that generate high-resolution images of tissue in a shorter assessment time than LDI. In addition, these devices provide the possibility to zoom in with increased resolution of a smaller area of interest. The moorFLPI-2 blood flow imager (Moor Instruments Ltd., Axminster, United Kingdom) and the PeriCam PSI (Perimed AB, Järfälla, Sweden) both use the laser speckle contrast technique to deliver real-time highresolution blood flow images. The latter system contains an invisible near infrared laser $(785 \mathrm{~nm})$, spreading the beam over the area of interest by a diffuser, creating a speckle pattern. Subsequently, blood perfusion is calculated by analyzing the variations (i.e., interference) in the speckle pattern. High values reflect high blood flow and should thereby indicate immature or hypertrophic scars.

A clinical study comparing the ability of LSPI with LDI in determining and monitoring hypertrophic scar perfusion shows a positive correlation $\left(r^{2}=0.86\right)$ [23] Moreover, in terms of feasibility, the LSPI device demonstrates a faster scan time and higher resolution, which may be an advantage for usage in clinical practice. Also, reactions to mechanical or pharmacological interventions can be studied nearly real time, allowing a dynamic way of studying scars. Perfusion rates within keloids are also assessed by LSI, showing significantly higher perfu- 
sion in keloids and adjacent skin compared with nonadjacent healthy skin [24]. However, a thorough clinimetric evaluation of LSI in scars is currently not available.

\subsection{Conclusion}

Color, elasticity, and perfusion are scar characteristics with a high clinical relevance. Two features, erythema and pigmentation, determine the color of the scar. Erythema is a complex characteristic that may be defined as the level of oxygenated hemoglobin measured at $660 \mathrm{~nm}$. Pigmentation is often referred to as the level of melanin, which can be excessively present (hyperpigmentation) or underrepresented (hypopigmentation). Reflectance spectroscopy is the most widely used measurement technique to assess scar color. Perfusion of vascularization and scar color are often bracketed together but both represent totally different features. The mixture of terms can also be found in literature where vascularization is described as scar color, blood flow, the presence of microvessels, as well as the amount of redness $[9,12,15,25]$. Conform definitions in several dictionaries and physiology textbooks, we would like to propose that vascularization is defined as the formation of blood vessels and capillaries in living tissue, which reflects a process. The presence of microvessels is a physical result of this process, and blood flow fulfils the functional role of perfusion. Scar redness is likely to be a derivative of all features that is assessed subjectively. For the purpose of clarity, it would be beneficial to provide a detailed description of every feature when assessed in research as well as in clinical practice, as we feel that the various scar features cannot be generalized into the umbrella term "vascularization." The term elasticity is most probably related to the lack of elastin in the dermal layer of scar tissue, but can also be considered as an umbrella term including extensibility, pliability, or suppleness of the scar. The most reliable assessment technique here measures vertical elasticity or extensibility by means of suction.

All tools are able to differentiate between scar tissue and healthy skin; however, this can also easily be determined by subjective evaluation. Objective tools should be able to monitor an individual patient in clinical practice, thereby distinguishing small scar changes. Thus, the measurement error of the tool must be smaller than the desired scar change to be measured. Taking into account reliability, patient friendliness, and feasibility in terms of cost and portability, a recommended panel of devices for the assessment of color, elasticity, and perfusion of scars consists of the DSM III ColorMeter for scar color, the Cutometer for scar elasticity and laser speckle imaging to assess scar perfusion.

\section{Take-Home Messages}

- A range of handheld tools is available to objectively assess physical scar parameters.

- When using a tool in clinical practice or for scientific use, take the clinimetric properties into account.

- To monitor scars in an individual patient, it is of paramount importance that the minimally important change (MIC) is smaller than the measurement error of the tool.

- It is showed that erythema and blood flow are not directly related to each other and therefore seem two different scar parameters (i.e., constructs).

- It is essential to provide a precise description of which scar parameter is aimed to assess.

- To assess scar color, the most widely used tool is the DSM III ColorMeter, offering read-out of erythema and melanin index values as well as CIE $\mathrm{L} * \mathrm{a} * \mathrm{~b}$ values.

- Elasticity can be best measured using the Cutometer, reflecting absolute and relative parameters of which Uf and Ue are recommended.

- To assess scar perfusion, laser Doppler imaging and laser Speckle imaging are available.

\section{References}

1. Mokkink LB, Terwee CB, Patrick DL, Alonso J, Stratford PW, Knol DL, et al. The COSMIN study reached international consensus on taxonomy, terminology, and definitions of measurement properties for health-related patient-reported outcomes. J Clin Epidemiol. 2010;63(7):737-45.

2. De Vet HCW, Terwee CB, Mokkink LB, Knol DL. Measurement in medicine. A practical guide. 1st ed. Cambridge: Cambridge Univ Press; 2011.

3. Crosby RD, Kolotkin RL, Williams GR. Defining clinically meaningful change in health-related quality of life. J Clin Epidemiol. 2003;56(5):395-407.

4. Mermans JF, Peeters WJ, Dikmans R, Serroyen J, van der Hulst RR, Van den Kerckhove E. A comparative study of colour and perfusion between two different post surgical scars. Do the laser Doppler imager and the colorimeter measure the same features of a scar? Skin Res Technol. 2013;19(2):107-14.

5. Jaspers MEH, Stekelenburg CM, Simons JM, Brouwer KM, Vlig M, van den Kerckhove E, et al. Assessing blood flow, microvasculature, erythema and redness in hypertrophic scars: a cross sectional study showing different features that require precise definitions. Burns. 2017;43(5):1044-50.

6. van der Wal M, Bloemen M, Verhaegen P, Tuinebreijer W, de Vet $\mathrm{H}$, van Zuijlen $\mathrm{P}$, et al. Objective color measurements: clinimetric performance of three devices on normal skin and scar tissue. $\mathrm{J}$ Burn Care Res. 2013;34(3):e187-94.

7. Chardon A, Cretois I, Hourseau C. Skin colour typology and suntanning pathways. Int J Cosmet Sci. 1991;13(4):191-208.

8. Van den Kerckhove E, Staes F, Flour M, Stappaerts K, Boeckx W. Reproducibility of repeated measurements on healthy skin with Minolta Chromameter CR-300. Skin Res Technol. 2001;7(1):56-9. 
9. Draaijers LJ, Tempelman FR, Botman YA, Kreis RW, Middelkoop E, van Zuijlen PP. Colour evaluation in scars: tristimulus colorimeter, narrow-band simple reflectance meter or subjective evaluation? Burns. 2004;30(2):103-7.

10. Deng H, Li-Tsang CWP. Measurement of vascularity in the scar: a systematic review. Burns. 2018;45(6):1253-65.

11. Nedelec B, Correa JA, Rachelska G, Armour A, LaSalle L. Quantitative measurement of hypertrophic scar: interrater reliability and concurrent validity. J Burn Care Res. 2008;29(3):501-11.

12. Verhaegen PD, van der Wal MB, Middelkoop E, van Zuijlen PP. Objective scar assessment tools: a clinimetric appraisal. Plast Reconstr Surg. 2011;127(4):1561-70.

13. Gankande TU, Duke JM, Wood FM, Wallace HJ. Interpretation of the DermaLab Combo(R) pigmentation and vascularity measurements in burn scar assessment: an exploratory analysis. Burns. 2015;41(6):1176-85.

14. Gankande TU, Duke JM, Danielsen PL, DeJong HM, Wood FM, Wallace HJ. Reliability of scar assessments performed with an integrated skin testing device - the DermaLab Combo((R)). Burns. 2014;40(8):1521-9.

15. Oliveira GV, Chinkes D, Mitchell C, Oliveras G, Hawkins HK, Herndon DN. Objective assessment of burn scar vascularity, erythema, pliability, thickness, and planimetry. Dermatol Surg. 2005;31(1):48-58.

16. van der Wal MB, Vloemans JF, Tuinebreijer WE, van de Ven $\mathrm{P}$, van Unen E, van Zuijlen PP, et al. Outcome after burns: an observational study on burn scar maturation and predictors for severe scarring. Wound Repair Regen. 2012;20(5):676-87.

17. Moncrieff M, Cotton S, Claridge E, Hall P. Spectrophotometric intracutaneous analysis: a new technique for imaging pigmented skin lesions. Br J Dermatol. 2002;146(3):448-57.
18. Draaijers LJ, Botman YA, Tempelman FR, Kreis RW, Middelkoop E, van Zuijlen PP. Skin elasticity meter or subjective evaluation in scars: a reliability assessment. Burns. 2004;30(2):109-14.

19. Corica GF, Wigger NC, Edgar DW, Wood FM, Carroll S. Objective measurement of scarring by multiple assessors: is the tissue tonometer a reliable option? J Burn Care Res. 2006;27(4):520-3.

20. Merkel PA, Silliman NP, Denton CP, Furst DE, Khanna D, Emery $\mathrm{P}$, et al. Validity, reliability, and feasibility of durometer measurements of scleroderma skin disease in a multicenter treatment trial. Arthritis Rheum. 2008;59(5):699-705.

21. Bray R, Forrester K, Leonard C, McArthur R, Tulip J, Lindsay R. Laser Doppler imaging of burn scars: a comparison of wavelength and scanning methods. Burns. 2003;29(3):199-206.

22. Ehrlich HP, Kelley SF. Hypertrophic scar: an interruption in the remodeling of repair--a laser Doppler blood flow study. Plast Reconstr Surg. 1992;90(6):993-8.

23. Stewart CJ, Frank R, Forrester KR, Tulip J, Lindsay R, Bray RC. A comparison of two laser-based methods for determination of burn scar perfusion: laser Doppler versus laser speckle imaging. Burns. 2005;31(6):744-52.

24. Liu Q, Wang X, Jia Y, Long X, Yu N, Wang Y, et al. Increased blood flow in keloids and adjacent skin revealed by laser speckle contrast imaging. Lasers Surg Med. 2016;48(4):360-4.

25. Brusselaers N, Pirayesh A, Hoeksema H, Verbelen J, Blot S, Monstrey S. Burn scar assessment: a systematic review of objective scar assessment tools. Burns. 2010;36(8):1157-64.

\section{Further Reading}

De Vet HCW, Terwee CB, Mokkink LB, Knol DL. Measurement in medicine. A practical guide. 1st ed. Cambridge: Cambridge Univ Press; 2011.

Open Access This chapter is licensed under the terms of the Creative Commons Attribution 4.0 International License (http://creativecommons. org/licenses/by/4.0/), which permits use, sharing, adaptation, distribution and reproduction in any medium or format, as long as you give appropriate credit to the original author(s) and the source, provide a link to the Creative Commons license and indicate if changes were made.

The images or other third party material in this chapter are included in the chapter's Creative Commons license, unless indicated otherwise in a credit line to the material. If material is not included in the chapter's Creative Commons license and your intended use is not permitted by statutory regulation or exceeds the permitted use, you will need to obtain permission directly from the copyright holder.

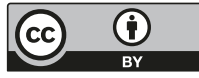

